\title{
Overexpression of DJ-1 and HSP90 $\alpha$, and loss of PTEN associated with invasive urothelial carcinoma of urinary bladder: Possible prognostic markers
}

\author{
HOJUNG LEE ${ }^{1}$, SEUNG KYU CHOI ${ }^{1}$ and JAE Y. RO ${ }^{2}$ \\ ${ }^{1}$ Department of Pathology, Eulji Medical Center, Eulji University School of Medicine, Seoul, Republic of Korea; \\ ${ }^{2}$ Department of Pathology, The Methodist Hospital, Weill Medical College of Cornell University, Houston, TX, USA
}

Received September 5, 2011; Accepted November 29, 2011

DOI: $10.3892 / \mathrm{ol} .2011 .522$

\begin{abstract}
DJ-1 and HSP90 $\alpha$ play a significant role in the progression of various types of cancer and are known to be associated with phosphatase and tensin homolog deleted on chromosome 10 (PTEN), PI3K-p110 $\alpha$ and pAkt, the signaling molecule proteins from the phosphatidylinositol 3-kinase (PI3K) pathway. However, the expression of these proteins and their clinical significance are not well characterized in urothelial carcinoma (UC). Immunohistochemical analysis of DJ-1, HSP90 $\alpha$, PTEN, pAkt and PI3K-p110 $\alpha$ expression was performed on tumor samples from 102 patients with UC to assess the relationship between the expression of each protein and the pathological parameters. The expression of DJ-1 and HSP90 $\alpha$ was positively correlated with the pathological stage of UC, whereas PTEN expression negatively correlated with, not only the pathological stage, but also the growth pattern and histological grade of UC. Although PI3K-p110 $\alpha$ expression was significantly correlated with DJ-1 as well as PTEN expression in UC, PI3K-p110 $\alpha$ expression itself failed to reveal any significant correlation with the clinicopathological parameters. In conclusion, the overexpression of DJ-1 and HSP90 $\alpha$, and a loss of PTEN are associated with invasive UC, and PI3K-p110 $\alpha$ expression is correlated with DJ-1 and PTEN expression in UC.
\end{abstract}

\section{Introduction}

Urothelial carcinoma (UC) is known to progress through two divergent pathways: one is the pathway characterized by superficial papillary tumors that harbor gain-of-function mutations of H-RAS, FGFR3 and phosphatidylinositol 3-kinase (PI3K), and the other is that of invasive tumors that exhibit defects in $\mathrm{p} 53$, retinoblastoma protein $(\mathrm{RB})$, and phosphatase and tensin homolog deleted on chromosome 10 (PTEN) (1).

Correspondence to: Professor Jae Y. Ro, Department of Pathology, The Methodist Hospital, Weill Medical College of Cornell University, 6565 Fannin Street, 77030 Houston, TX, USA

E-mail: jaero@tmhs.org

Key words: DJ-1, HSP90a, PTEN, PI3K-p110a, pAkt, urothelial carcinoma
PTEN is a key negative regulator of the PI3K-protein kinase $\mathrm{B}(\mathrm{PKB} / \mathrm{Akt})$ signaling pathway (2). PI3K, activated by growth factors, catalyzes the phosphorylation of phosphatidylinositol $(4,5)$-biphosphate (PIP2) to phosphatidylinositol (3,4,5)-triphosphate (PIP3). PIP3 recruits 3-phosphoinositidedependent kinase (PDK), which phosphorylates and activates Akt (3). Akt is a signal transduction protein that plays a key role in multiple signaling pathways, including cell proliferation, apoptosis and transcription (4). Loss of PTEN expression results in an increased concentration of PIP3 and causes Akt to transform into its phosphorylated, active form (pAkt) $(2,5)$.

Heat shock protein 90 (HSP90) is a molecular chaperone required for the stability and function of various client proteins, which mediate a major property necessary for the transformation of a normal cell to a cancer cell $(6,7)$. HSP90 has two isoforms, HSP90 $\alpha$ and HSP90 $\beta$, but it has been reported that only HSP90 $\alpha$ is capable of activating oncogenic kinases (8). Inhibition of HSP90 induces the degradation and inactivation of a number of HSP90 client proteins, including protein kinases [human epidermal growth factor receptor 2 (HER2) and $\mathrm{Akt}$, steroid hormone receptors and mutant oncoproteins (mutant p53 and B-Raf), leading to an antitumor effect $(9,10)$.

DJ-1 is a conserved protein, coded by the gene Parkinson disease 7 (PARK7). It is associated with an autosomal recessive early-onset Parkinson's disease (PD) (11). This protein is ubiquitously present in cells and is involved in diverse cell processes such as cell transformation, control of protein-RNA interaction and preventing cell death from oxidative stress-induced apoptosis (12). DJ-1 promotes cell survival by modulating PTEN (13), and high DJ-1 levels have been reported during initiation and progression in certain types of cancer (14-16).

The alterations of PTEN, pAkt and PI3K have been found to contribute to bladder carcinogenesis $(1,17,18)$. However, DJ-1 and HSP90 $\alpha$ expression in bladder tumors compared with clinicopathological parameters has yet to be studied, although total HSP90 expression in bladder tumors has rarely been described $(19,20)$.

In the present study, we analyzed the expression of DJ-1, HSP90 $\alpha$, PTEN, pAkt and PI3K-p110 $\alpha$ (p110 $\alpha$ subunit of $\mathrm{PI} 3 \mathrm{~K}$ ) in bladder tumors and their association with pathological parameters to determine the role of these proteins in the prognostic significance of UC. 


\section{Materials and methods}

Patients and samples. Tissue samples from 102 UC patients who underwent transurethral resection of bladder tumor (88 cases) or radical cystectomy (14 cases) at the Eulji Medical Center, Eulji University School of Medicine (Seoul, Korea), between 2004 and 2008, were enrolled in this study. The pathology slides were reviewed, and representative sections of each tumor and tumor-free bladder tissues, considered as 'normal', from the 14 radical cystectomy specimens were selected as a control. The histological grade of the bladder tumors was determined according to the 2004 World Health OrganizationInternational Society of Urological Pathology (WHO-ISUP) classification (21). Table I shows the clinical and pathological data of patients. Following the review of all 102 UC sections, the most representative areas of tumor tissues $(2 \mathrm{~mm}$ in diameter) were removed from the paraffin blocks and arranged in a new recipient block for tissue microarray (TMA). TMAs were constructed using a microarray instrument (Unitma Co., Seoul, Korea). The institutional review board of Eulji Medical Center approved this study.

Immunohistochemistry. Immunohistochemical staining was performed using Dako Autostainer (DakoCytomation, Carpinteria, CA, USA). Tissue sections ( $4-\mu \mathrm{m})$ were obtained from TMA blocks and transferred onto poly-L-lysine-coated slides. Following deparaffinization and rehydration, antigen retrieval was performed using citrate buffer $(\mathrm{pH} 6.0)$ at $121^{\circ} \mathrm{C}$ for $10 \mathrm{~min}$. Endogenous peroxidase activity was blocked with $3 \%$ hydrogen peroxide for $5 \mathrm{~min}$, and the sections were incubated with antibodies against DJ-1 (Abcam, Cambridge, UK; 1:1,000), HSP90 $\alpha$ (Abcam; 1:10,000), pAkt (Abcam; 1:500), PI3K-p110 $\alpha$ (Cell Signaling, Danvers, MA, USA; 1:500) and PTEN (Epitomics, Burlingame, CA, USA; 1:250). Color was developed using diaminobenzidine, and the slides were counterstained with hematoxylin. Normal urinary bladder mucosa was used as a positive control for DJ-1, HSP90 $\alpha$ and PTEN. Endothelial cells were used as positive controls for PI3K-p110 $\alpha$ and breast carcinoma was used as a positive control for pAkt. Cases not treated with primary antibodies served as negative controls.

Immunohistochemical scoring of all antibodies used for this study was based on the intensity of staining from the most intensely stained area. The staining intensity was scored as: 0 , no expression; $1+$, low expression (weak intensity); and 2+, high expression (moderate to strong intensity) (Fig. 1).

Statistical analysis. The Chi-square test and Fisher's exact test were used to evaluate the correlation between the immunoreactivity of DJ-1, HSP $90 \alpha$, PTEN, pAkt and PI3K-p110 $\alpha$, and clinicopathological characteristics. The Spearman's rank correlation test was used to analyze the association between markers. $\mathrm{P}<0.05$ was considered to indicate a statistically significant difference. The analyses were performed using the IBM SPSS version 19.0.

\section{Results}

Staining patterns of DJ-1, PTEN, PI3K-p110 $\alpha$, pAkt and HSP90 $\alpha$ in normal urothelium and UC with varying intensity
Table I. Patient and tumor characteristics.

\begin{tabular}{lll}
\hline Characteristics & \multicolumn{2}{c}{ Number of patients } \\
\cline { 2 - 3 } & $\mathrm{n}$ & $(\%)$ \\
\hline Age & & \\
$<70$ & 46 & 45.1 \\
$\geq 70$ & 56 & 54.9 \\
Gender & & \\
Male & 83 & 81.4 \\
Female & 19 & 18.6 \\
Growth pattern & & \\
Papillary & 66 & 64.7 \\
Non-papillary & 36 & 35.3 \\
Tumor stage & & \\
Ta & 34 & 33.3 \\
T1 & 40 & 39.2 \\
T2 & 16 & 15.7 \\
T3 & 12 & 11.8 \\
Tumor grade & & \\
Low & 51 & 50 \\
High & 51 & 50 \\
\hline
\end{tabular}

Ta, non-invasive tumors.

are shown in Fig. 1.DJ-1 in the normal urothelium was localized in the nuclei, cytoplasm and cell membrane in all cases. DJ-1 expression in UC was mostly cytoplasmic with some nuclear and membranous staining. DJ-1 was expressed in $84.3 \%$ (low $17.6 \%$ and high expression $66.7 \%$ ) of UCs. HSP90 $\alpha$ exhibited cytoplasmic and membranous localization in normal urothelial cells and in $91.2 \%$ of UCs (low $25.5 \%$ and high expression $65.7 \%$ ). A strong expression of DJ-1 and HSP90 $\alpha$ was observed more in non-papillary, high-grade, invasive UCs compared to papillary, low-grade and non-invasive ones (Fig. 1). PTEN expression was observed in the cytoplasm and nuclei of normal urothelial cells in all cases and $63.7 \%$ of UCs (low $42.2 \%$ and high expression 21.5\%). In contrast to DJ-1 and HSP90 $\alpha$ expression, PTEN expression was much stronger in papillary, non-invasive and low-grade UCs compared to non-papillary, high-grade, invasive UCs (Fig. 1). PI3K-p110 $\alpha$ exhibited only membranous staining in the normal urothelium, but membranous and cytoplasmic staining in UC. PI3K-p $110 \alpha$ was expressed in $67.6 \%$ (low $41.2 \%$ and high expression $26.4 \%$ ) of UC cases. pAkt expression was heterogeneous with speckled cytoplasmic and membranous staining in normal urothelium and UCs. pAkt was expressed in $64.7 \%$ (low $32.35 \%$ and high expression $32.35 \%$ ) of UCs.

Table II shows the correlation between markers analyzed and clinically important histopathological features of the tumors. The expression of DJ-1 and HSP90 $\alpha$ was positively correlated with the invasiveness of the tumor, with a greater expression in T1-T3 tumors compared to non-invasive tumors (Ta) [Spearman's correlation coefficient (cc), 0.201; $\mathrm{P}=0.043$ and cc, $0.303 ; \mathrm{P}=0.002$, respectively], but not with growth 
Table II. Correlation between histopathological variables and expression of each protein.

\begin{tabular}{|c|c|c|c|c|c|}
\hline & DJ-1 & PTEN & PI3K-p $110 \alpha$ & $\mathrm{pAkt}$ & HSP90 $\alpha$ \\
\hline Non-papillary & $0.790^{\mathrm{a}}$ & $0.001^{\mathrm{a}}$ & $0.274^{\mathrm{a}}$ & 0.451 & 0.184 \\
\hline$\geq \mathrm{T} 1$ & 0.043 & $0.013^{a}$ & 0.422 & 0.078 & 0.002 \\
\hline High grade & 0.632 & $<0.001^{\mathrm{a}}$ & 0.832 & 0.120 & 0.081 \\
\hline DJ-1 & - & 0.072 & $<0.001$ & 0.872 & 0.170 \\
\hline PTEN & & - & 0.014 & 0.375 & $0.141^{\mathrm{a}}$ \\
\hline PI3K-p110 $\alpha$ & & & - & 0.055 & 0.569 \\
\hline pAkt & & & & - & 0.495 \\
\hline $\operatorname{HSP} 90 \alpha$ & & & & & - \\
\hline
\end{tabular}

${ }^{a}$ Negative correlation. $\mathrm{P}<0.05$ (shown in bold) denotes statistically significant differences between variable vs. protein expression.

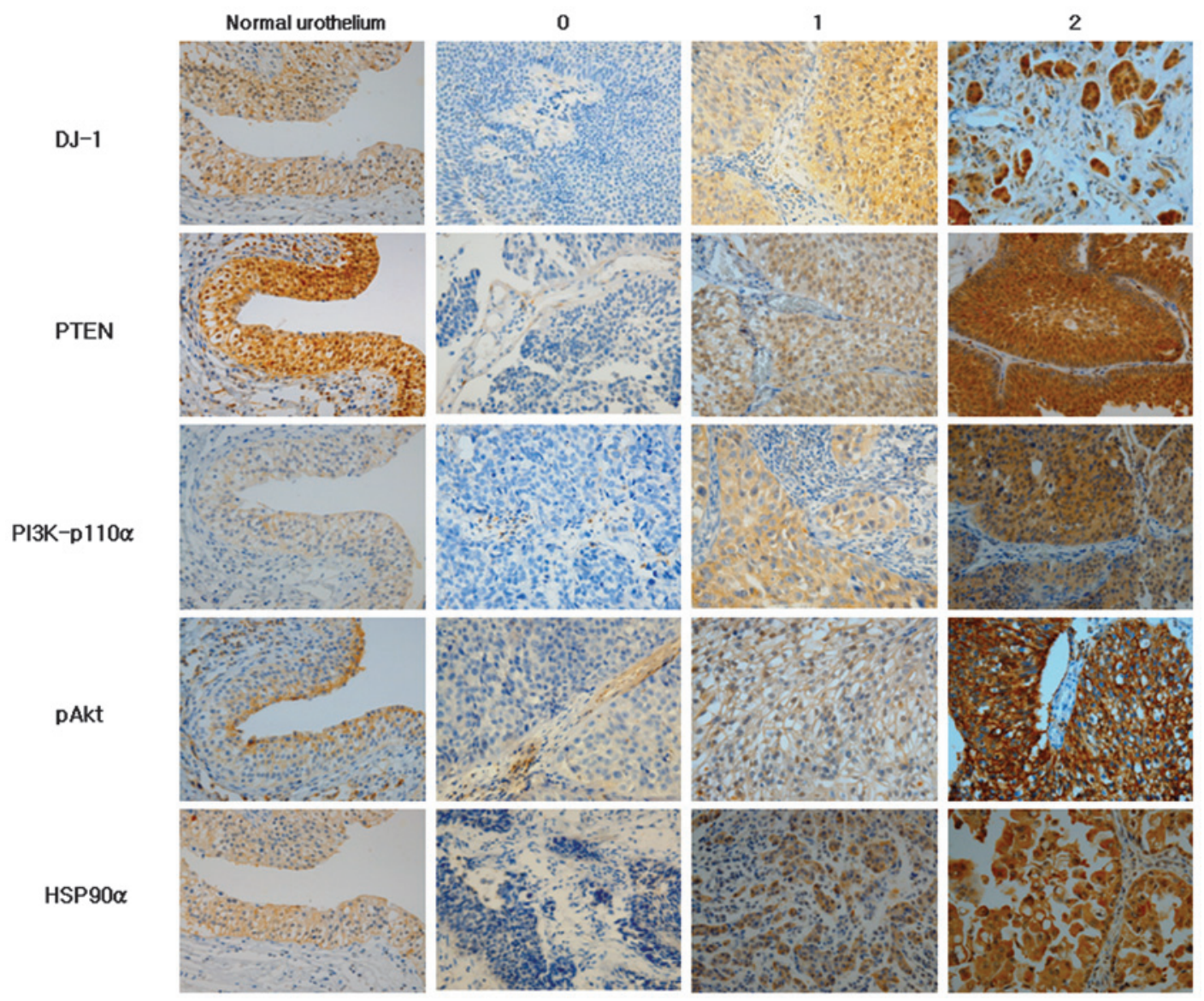

Figure 1. Immunohistochemical staining of DJ-1, PTEN, PI3K-p110 $\alpha$, pAkt and HSP90 $\alpha$ in normal urothelium and urothelial carcinoma according to staining intensity.

pattern (papillary vs. non-papillary) and histological grade. A significant negative correlation was observed between PTEN expression and non-papillary growth pattern (cc, -0.324; $\mathrm{P}=0.001)$, invasive tumor (cc, $-0.246 ; \mathrm{P}=0.013)$ and a high histological grade (cc, $-0.365 ; \mathrm{P}<0.001$ ). However, PI3K-p110 $\alpha$ and pAkt expression did not correlate with any histopathological parameters. A statistically significant positive correlation was present between DJ-1 and PI3K-p110 $\alpha$ expression (cc, 0.367; $<0.001)$ as well as between PI3K-p110 $\alpha$ and PTEN expression (cc, $0.242 ; \mathrm{P}=0.014$ ) in UC. Table III shows the positive correla- tion between the markers in a two by two comparison using the Chi-square test. The majority of the PI3K-p110 $\alpha$-positive UCs also stained positively for DJ-1 $(64 / 69,92.7 \%)$. Of 33 negative samples for PI3K-p110 $\alpha, 22$ cases $(66.7 \%)$ were DJ-1-positive (Table IIIA). PTEN-positive tumors were more common in PI3K-p110 $\alpha$-positive samples (71\%, 49/69 cases) compared to PI3K-p110 $\alpha$-negative samples (48.5\%, 16/33 cases; Table IIIB). No significant correlation was found among other markers.

The histopathological characteristics associated with DJ-1, PTEN and HSP90 $\alpha$ staining intensity are shown in Table IV. 
Table III. Two by two comparisons of PI3K-p110 $\alpha$ and DJ-1, and PI3K-p110 $\alpha$ and PTEN.

A, Comparison of PI3K-p110 $\alpha$ and DJ-1 ${ }^{\mathrm{a}}$.

\begin{tabular}{lrrr}
\hline PI3K-p110 $\alpha$ & - & + & Total \\
\hline DJ-1 & & & \\
- & 11 & 5 & 16 \\
+ & 22 & 64 & 86 \\
Total & 33 & 69 & 102 \\
\hline
\end{tabular}

B, Comparison of PI3K-p110 $\alpha$ and PTEN ${ }^{b}$.

\begin{tabular}{lllr}
\hline PI3K-p110 $\alpha$ & - & + & Total \\
\hline PTEN & & & \\
- & 17 & 20 & 37 \\
+ & 16 & 49 & 65 \\
Total & 33 & 69 & 102 \\
\hline
\end{tabular}

${ }^{\mathrm{a}} \mathrm{P}=0.002,{ }^{\mathrm{b}} \mathrm{P}=0.031$.

The higher expression (score 2) of DJ-1 and HSP90 $\alpha$ was significantly associated with invasive tumors $(\mathrm{P}=0.026$ and $\mathrm{P}=0.004$, respectively) compared to non-invasive tumors (Ta). The loss of PTEN expression (score 0) was significantly correlated with non-papillary, invasive and high-grade tumors $(\mathrm{P}=0.005, \mathrm{P}=0.034$ and $\mathrm{P}<0.001$, respectively).

\section{Discussion}

DJ-1 improves tumor cell survival by modulating the Akt/PI3K axis $(13,14)$, inhibiting apoptosis through repression of the p53-Bax-caspase pathway (22) and stabilizing the antioxidant transcriptional master regulator Nrf2 (23). Downregulation of DJ-1 by transfection with small interfering RNA (siRNA) targets DJ-1 inhibited cell proliferation and enhances apoptosis of laryngeal squamous cell carcinoma (SCC) Hep-2 cells (24). DJ-1 has been reported to be overexpressed in several types of human cancer, including lung, breast, pancreas and esophageal (13-16). However, DJ-1 expression status in bladder tumors has not yet been reported. In the present study, a high expression of DJ-1 was detected in invasive tumors (T1-T3) compared to non-invasive tumors (Ta) of the bladder. This result supports previous findings that high levels of DJ-1 expression are associated with a higher pathological tumor stage in SCCs of the esophagus and glottis $(14,24)$. These studies report that a higher expression of DJ-1 was observed in pT3-pT4 glottic SCCs compared with pTis-pT2 tumors (24) and in T4 esophageal SCCs compared with T1-T3 tumors (14). Taken together, these results indicate that a high expression of DJ-1 is associated with tumor invasiveness in carcinomas.

Deletion of PTEN or reduced PTEN expression has been well established in invasive UC $(17,25,26)$. A strong expression of PTEN observed in the nucleus and cytoplasm in normal urothelium was lost or reduced in the nucleus and cytoplasm in UCs in our study, which was in concordance with findings from previous studies $(17,27)$. Loss of PTEN expression was significantly associated with non-papillary, invasive and high-grade tumors. Similarly, the proportion of strong PTEN expression positive cases was lower in non-papillary, invasive and high-grade tumors compared to papillary, non-invasive and low-grade tumors.

Mutation of PI3K-p110 $\alpha$, a catalytic subunit of PI3K, has been found in a significant proportion of low-grade and low stage UCs (28). In another study, PI3K-p110 $\alpha$ mutation was detected in $25 \%$ of UC samples and $26 \%$ of UC-derived cell lines with mutations in a helical domain (29). PI3K-p110 $\alpha$ was weakly expressed in the membrane of normal urothelium and in the cytoplasm or membrane of UC tumor cells, but showed no difference in immunoreactivity according to the growth pattern, grade and stage of UC in our study.

An activating mutation of Akt was identified in renal pelvic UC (30) as well as renal cell carcinoma (31) and prostatic adenocarcinoma (32), but not in urinary bladder UC (33). In bladder UC, pAkt loss was described in invasive UC compared to benign urothelium and non-invasive UC $(26,27)$. In the present study, pAkt was heterogeneously stained in the cytoplasm and membrane of normal urothelium as well as UC tumor cells. However, a significant loss or overexpression of pAkt was not observed in UC according to the pathological characteristics.

HSP90 overexpression is known to be correlated with the evolution of a number of tumors, and HSP90 inhibitors, such as geldanamycin derivatives, are currently undergoing clinical trials for the treatment of advanced carcinomas of the breast, lung, colon and esophagus, as well as melanoma and sarcomas (34-36). In cases of UC, 17-allylamino-17-demethoxygeldanamycin (17-AAG) was applied to human urinary bladder cancer cell lines that resulted in cell cycle arrest and apoptosis by inducing the downregulation of HSP90 client proteins (37). HSP90 expression in UC is not as well understood as in other tumors. Cardillo et al (20) reported that a higher level of HSP90 expression was shown in high-grade and muscle-invasive UCs than in low-grade and superficial UCs. Contrary to this result, Lebret et al (19) demonstrated that a loss of HSP90 expression was associated with the risk of developing an infiltrating recurrence of UC. However, in their study, the sample size of infiltrating recurrent tumors was limited to 5 cases and the difference of pathological features according to the intensity of HSP90 expression was not mentioned. Differential expression of $\alpha$ and $\beta$ isoforms of HSP90 was studied in gastrointestinal stromal tumor (GIST), and it appears that HSP90 $\alpha$ is more relevant to the intrinsic aggressiveness of GIST (38). Initially, we used HSP90 $\alpha$ and HSP90 $\beta$ antibodies; however, HSP90 $\beta$ was not detected in any normal urothelium or UC samples, thus HSP90 $\beta$ was excluded from this study (data not shown). In the present study, the expression of HSP90 $\alpha$ was higher in invasive (T1-T3) compared to non-invasive tumors (Ta), suggesting that a higher level of HSP90 $\alpha$ is linked to invasiveness in UC and supports the potential use of HSP90 inhibitors as an adjunctive therapeutic agent in invasive UC.

The interaction between DJ-1 and the PI3K/Akt pathway genes was studied in experiments using Drosophila as a model system. Yang et al (39) inhibited the function of a Drosophila DJ-1 homologue (DJ-1A) by transgenic RNA interference (RNAi) and DJ-1A RNAi flies exhibited an accumulation 
Table IV. Histopathological characteristics of DJ-1, PTEN and HSP90 $\alpha$ staining intensity.

\begin{tabular}{|c|c|c|c|c|c|c|}
\hline Marker & Score & $0(\%)$ & $1(\%)$ & $2(\%)$ & Total & P-value \\
\hline \multicolumn{7}{|l|}{ DJ-1 } \\
\hline Growth pattern & & & & & & 0.657 \\
\hline Papillary & & $11(16.7)$ & $10(15.1)$ & $45(68.2)$ & 66 & \\
\hline Non-papillary & & $5(13.9)$ & $8(22.2)$ & $23(63.9)$ & 36 & \\
\hline Stage & & & & & & 0.026 \\
\hline $\mathrm{Ta}$ & & $10(29.4)$ & $5(14.7)$ & $19(55.9)$ & 34 & \\
\hline T1-T3 & & $6(8.8)$ & $13(19.1)$ & $49(72.1)$ & 68 & \\
\hline Histological grade & & & & & & 0.857 \\
\hline Low grade & & $9(17.6)$ & $9(17.6)$ & $33(64.8)$ & 51 & \\
\hline High grade & & $7(13.7)$ & $9(17.6)$ & $35(68.7)$ & 51 & \\
\hline \multicolumn{7}{|l|}{ PTEN } \\
\hline Growth pattern & & & & & & 0.005 \\
\hline Papillary & & $17(25.8)$ & $30(45.5)$ & $19(28.8)$ & 66 & \\
\hline Non-papillary & & $20(55.6)$ & $13(36.1)$ & $3(8.3)$ & 36 & \\
\hline Stage & & & & & & 0.034 \\
\hline $\mathrm{Ta}$ & & $8(23.5)$ & $14(41.2)$ & $12(35.3)$ & 34 & \\
\hline T1-T3 & & $29(42.6)$ & $29(42.6)$ & $10(14.7)$ & 68 & \\
\hline Histological grade & & & & & & $<0.001$ \\
\hline Low grade & & $9(17.6)$ & $27(52.9)$ & $15(29.4)$ & 51 & \\
\hline High grade & & $28(54.9)$ & $16(31.4)$ & $7(13.7)$ & 51 & \\
\hline \multicolumn{7}{|l|}{ HSP90 $\alpha$} \\
\hline Growth pattern & & & & & & 0.294 \\
\hline Papillary & & $6(9.1)$ & $20(30.3)$ & $40(60.6)$ & 66 & \\
\hline Non-papillary & & $3(8.3)$ & $6(16.7)$ & $27(75.0)$ & 36 & \\
\hline Stage & & & & & & 0.004 \\
\hline $\mathrm{Ta}$ & & $4(11.8)$ & $15(44.1)$ & $15(44.1)$ & 34 & \\
\hline T1-T3 & & $5(7.4)$ & $11(16.2)$ & $52(76.5)$ & 68 & \\
\hline Histological grade & & & & & & 0.151 \\
\hline Low grade & & $5(9.8)$ & $17(33.3)$ & $29(56.9)$ & 51 & \\
\hline High grade & & $4(7.8)$ & $9(17.6)$ & $38(74.5)$ & 51 & \\
\hline
\end{tabular}

$\mathrm{P}<0.05$ (shown in bold) denotes statistically significant difference in histopathological characteristic vs. staining intensity. Ta, non-invasive tumors.

of reactive oxygen species, photoreceptor neuronal loss and eye degeneration. An enhancement of eye degeneration was observed when PTEN was coexpressed with the DJ-1A RNAi transgene, whereas a clear suppression of the DJ-1A RNAi phenotype was observed and the eyes were restored to normal size when the wild-type form of the PI3K catalytic subunit Dp110 was coexpressed. These authors also found reduced phosphorylation of Akt in DJ-1A RNAi animals, suggesting an impairment of PI3K/Akt signaling by DJ-1A downregulation. Our data showed a positive correlation between DJ-1 and PI3K-p110 $\alpha$ expression, which concurred with the findings from the study by Yang et al (39), although the data did not show a negative correlation between the expression of DJ-1 and PTEN, nor a positive correlation between the expression of DJ-1 and pAkt. A positive correlation between the expression of PI3K-p110 $\alpha$ and PTEN in our data was noteworthy as PTEN exerts enzymatic activity as a PIP3 phosphatase, opposing the activity of PI3K (40). PTEN-inactivating and $\mathrm{PI} 3 \mathrm{~K}$-activating mutations is known to coexist in endometrial, breast and colorectal cancer (41). In UC, loss of PTEN has been associated with invasive behavior and the mutation of PI3K has been reported in low-grade papillary tumors. Our finding that $71 \%$ of PI3K-p110 $\alpha$-positive samples stained positively for PTEN suggests that alteration of these proteins may have non-canonical effects on the development of UC, as Platt et al (29) previously proposed. Platt et al demonstrated that overexpression of PI3K-p110 $\alpha$ does not necessarily involve amplification of the gene. Moreover, no significant correlation was found between the alteration of PI3K pathway members PI3K-p110 $\alpha$, TSC1 and PTEN, suggesting that the mutation of these members may be independently distributed in UC.

In conclusion, findings of our study have shown that the overexpression of DJ-1 and HSP90 $\alpha$ is associated with invasiveness of UC, and that loss of PTEN is associated with 
non-papillary histology, high-grade and invasive UCs. The expression of PI3K-p110 $\alpha$ is correlated with DJ-1 and PTEN expression in UC. However, a correlation for DJ-1, HSP90 $\alpha$ and PTEN expression was not observed. Additional studies through mutational analysis of these markers are required to better understand their contribution to bladder tumorigenesis and molecular prognostic markers.

\section{Acknowledgements}

We thank Deoksu Kim for technical support including tissue microarray construction and immunohistochemistry.

\section{References}

1. Castillo-Martin M, Domingo-Domenech J, Karni-Schmidt O, Matos T and Cordon-Cardo C: Molecular pathways of urothelial development and bladder tumorigenesis. Urol Oncol 28: 401-408, 2010.

2. Morgensztern D and McLeod HL: PI3K/Akt/mTOR pathway as a target for cancer therapy. Anticancer Drugs 16: 797-803, 2005.

3. Alessi DR, James SR, Downes CP, et al: Characterization of a 3-phosphoinositide-dependent protein kinase which phosphorylates and activates protein kinase B alpha. Curr Biol 7: 261-269, 1997.

4. Song G, Ouyang $\mathrm{G}$ and Bao S: The activation of Akt/PKB signaling pathway and cell survival. J Cell Mol Med 9: 59-71, 2005.

5. Di Cristofano A and Pandolfi PP: The multiple roles of PTEN in tumor suppression. Cell 100: 387-390, 2000.

6. Whitesell L and Lindquist SL: HSP90 and the chaperoning of cancer. Nat Rev Cancer 5: 761-772, 2005.

7. $\mathrm{Xu} \mathrm{W}$ and Neckers L: Targeting the molecular chaperone heat shock protein 90 provides a multifaceted effect on diverse cell signaling pathways of cancer cells. Clin Cancer Res 13: 1625-1629, 2007.

8. Millson SH, Truman AW, Racz A, et al: Expressed as the sole Hsp90 of yeast, the alpha and beta isoforms of human Hsp90 differ with regard to their capacities for activation of certain client proteins, whereas only Hsp90beta generates sensitivity to the Hsp90 inhibitor radicicol. FEBS J 274: 4453-4463, 2007.

9. Solit DB,Ivy SP,Kopil C, et al: Phase I trial of 17-allylamino-17-demethoxygeldanamycin in patients with advanced cancer. Clin Cancer Res 13: 1775-1782, 2007.

10. Basso AD, Solit DB, Chiosis G, Giri B, Tsichlis P and Rosen N: Akt forms an intracellular complex with heat shock protein 90 (Hsp90) and Cdc37 and is destabilized by inhibitors of Hsp90 function. J Biol Chem 277: 39858-39866, 2002.

11. Bonifati V, Rizzu P, Squitieri F, et al: DJ-1 (PARK7), a novel gene for autosomal recessive, early onset parkinsonism. Neurol Sci 24: 159-160, 2003.

12. Taira T, Saito Y, Niki T, Iguchi-Ariga SM, Takahashi K and Ariga H: DJ-1 has a role in antioxidative stress to prevent cell death. EMBO Rep 5: 213-218, 2004.

13. Kim RH, Peters M, Jang Y, et al: DJ-1, a novel regulator of the tumor suppressor PTEN. Cancer Cell 7: 263-273, 2005.

14. Yuen HF, Chan YP, Law S, et al: DJ-1 could predict worse prognosis in esophageal squamous cell carcinoma. Cancer Epidemiol Biomarkers Prev 17: 3593-3602, 2008.

15. Le Naour F, Misek DE, Krause MC, et al: Proteomics-based identification of RS/DJ-1 as a novel circulating tumor antigen in breast cancer. Clin Cancer Res 7: 3328-3335, 2001.

16. Tian M, Cui YZ, Song GH, et al: Proteomic analysis identifies MMP-9, DJ-1 and A1BG as overexpressed proteins in pancreatic juice from pancreatic ductal adenocarcinoma patients. BMC Cancer 8: 241, 2008.

17. Knowles MA, Platt FM, Ross RL and Hurst CD: Phosphatidylinositol 3-kinase (PI3K) pathway activation in bladder cancer. Cancer Metastasis Rev 28: 305-316, 2009.

18. Askham JM, Platt F, Chambers PA, Snowden H, Taylor CF and Knowles MA: AKT1 mutations in bladder cancer: identification of a novel oncogenic mutation that can co-operate with E17K. Oncogene 29: 150-155, 2010.

19. Lebret T, Watson RW, Molinie V, et al: Heat shock proteins HSP27, HSP60, HSP70, and HSP90: expression in bladder carcinoma. Cancer 98: 970-977, 2003.
20. Cardillo MR, Sale P and Di Silverio F: Heat shock protein-90, IL-6 and IL-10 in bladder cancer. Anticancer Res 20: 4579-4583, 2000.

21. Ebele JN, Sauter G, Epstein JI and Sesterhenn IA (eds): World Health Organization Classification of Tumors. Pathology and Genetics of Tumours of the Urinary System and Male Genital Organs. IARC Press, Lyon, 2004.

22. Fan J, Ren H, Jia N, et al: DJ-1 decreases Bax expression through repressing p53 transcriptional activity. J Biol Chem 283: 4022-4030, 2008.

23. Clements CM, McNally RS, Conti BJ, Mak TW and Ting JP: DJ-1, a cancer- and Parkinson's disease-associated protein, stabilizes the antioxidant transcriptional master regulator Nrf2. Proc Natl Acad Sci USA 103: 15091-15096, 2006.

24. Zhu XL, Wang ZF, Lei WB, Zhuang HW, Jiang HY and Wen WP: DJ-1: A novel independent prognostic marker for survival in glottic squamous cell carcinoma. Cancer Sci 101: 1320-1325, 2010.

25. Puzio-Kuter AM, Castillo-Martin M, Kinkade CW, et al: Inactivation of $\mathrm{p} 53$ and Pten promotes invasive bladder cancer. Genes Dev 23: 675-680, 2009.

26. Harris LD, De La Cerda J, Tuziak T, et al: Analysis of the expression of biomarkers in urinary bladder cancer using a tissue microarray. Mol Carcinog 47: 678-685, 2008.

27. Schultz L, Albadine R, Hicks J, et al: Expression status and prognostic significance of mammalian target of rapamycin pathway members in urothelial carcinoma of urinary bladder after cystectomy. Cancer 116: 5517-5526, 2010.

28. Lopez-Knowles E, Hernandez S, Malats N, et al: PIK3CA mutations are an early genetic alteration associated with FGFR3 mutations in superficial papillary bladder tumors. Cancer Res 66: 7401-7404, 2006.

29. Platt FM, Hurst CD, Taylor CF, Gregory WM, Harnden P and Knowles MA: Spectrum of phosphatidylinositol 3-kinase pathway gene alterations in bladder cancer. Clin Cancer Res 15: 6008-6017, 2009.

30. Qian CN, Furge KA, Knol J, et al: Activation of the PI3K/AKT pathway induces urothelial carcinoma of the renal pelvis: identification in human tumors and confirmation in animal models. Cancer Res 69: 8256-8264, 2009.

31. Pantuck AJ, Seligson DB, Klatte T, et al: Prognostic relevance of the mTOR pathway in renal cell carcinoma: implications for molecular patient selection for targeted therapy. Cancer 109: 2257-2267, 2007.

32. King JC, Xu J, Wongvipat J, et al: Cooperativity of TMPRSS2-ERG with PI3-kinase pathway activation in prostate oncogenesis. Nat Genet 41: 524-526, 2009.

33. Yoo LI, Liu DW, Le Vu S, Bronson RT, Wu H and Yuan J: Pten deficiency activates distinct downstream signaling pathways in a tissue-specific manner. Cancer Res 66: 1929-1939, 2006.

34. Ramanathan RK, Egorin MJ, Erlichman C, et al: Phase I pharmacokinetic and pharmacodynamic study of 17-dimethylaminoethylamino-17-demethoxygeldanamycin, an inhibitor of heat-shock protein 90, in patients with advanced solid tumors. J Clin Oncol 28: 1520-1526, 2010.

35. Modi S, Stopeck A, Linden H, et al: HSP90 inhibition is effective in breast cancer: a phase II trial of tanespimycin (17-AAG) plus trastuzumab in patients with HER2-positive metastatic breast cancer progressing on trastuzumab. Clin Cancer Res 17: 5132-5139, 2011.

36. Pacey S, Wilson RH, Walton M, et al: A phase I study of the heat shock protein 90 inhibitor alvespimycin (17-DMAG) given intravenously to patients with advanced solid tumors. Clin Cancer Res 17: 1561-1570, 2011.

37. Karkoulis PK, Stravopodis DJ, Margaritis LH and Voutsinas GE: 17-Allylamino-17-demethoxygeldanamycin induces downregulation of critical Hsp90 protein clients and results in cell cycle arrest and apoptosis of human urinary bladder cancer cells. BMC Cancer 10: 481, 2010.

38. Li CF, Huang WW, Wu JM, et al: Heat shock protein 90 overexpression independently predicts inferior disease-free survival with differential expression of the alpha and beta isoforms in gastrointestinal stromal tumors. Clin Cancer Res 14: 7822-7831, 2008.

39. Yang Y, Gehrke S, Haque ME, et al: Inactivation of Drosophila DJ-1 leads to impairments of oxidative stress response and phosphatidylinositol 3-kinase/Akt signaling. Proc Natl Acad Sci USA 102: 13670-13675, 2005.

40. Georgescu MM: PTEN tumor suppressor network in PI3K-Akt pathway control. Genes Cancer 1: 1170-1177, 2010.

41. Yuan TL and Cantley LC: PI3K pathway alterations in cancer: variations on a theme. Oncogene 27: 5497-5510, 2008. 